\title{
Tendencia espacio-temporal de clasificación BIRADS sugestiva de malignidad: un análisis nacional de mastografías, 2013-2017
}

Nancy E. Navarro-Ruíz y Antonio Reyna-Sevilla*

Departamento de Salud Pública, Centro Universitario de Ciencias de la Salud, Universidad de Guadalajara, Guadalajara, Jal., México

\section{Resumen}

Introducción: Promover la detección de cáncer de mama (CaMa) en mujeres mediante mastografía es una estrategia viable para disminuir los diagnósticos en fases clínicamente avanzadas y la mortalidad. Objetivos: Describir los resultados reportados por estudios de mastografía en mujeres realizados a nivel nacional durante 2013-2017 y analizar la tendencia espaciotemporal de categorías BIRADS (Breast Imaging Reporting and Data System) sugestivas de malignidad por Estado. Método: Diseño analítico longitudinal que incluyó información sobre estudios de mastografía de mujeres según grupo de edad (<40 e $\geq 40$ ), valoradas en unidades de la Secretaría de Salud, México, durante 2013-2017. Se estimó la frecuencia de categorías según BIRADS, tasa estandarizada sugestiva de malignidad (categorías 4 y 5) en mujeres $\geq 40$ años y se utilizó estadística espacial para analizar la tendencia por Estado. Resultados: Se analizaron 3,659,151 mastografías, el $98.5 \%$ en mujeres $\geq 40$ años. La tasa sugestiva de malignidad disminuyó de 38.3 (2013) a 31 (2017) por 100 mil mujeres $\geq 40$ años; sin embargo, el riesgo de detección aumentó hasta 13 veces en diez Estados. Conclusiones: Aunque el riesgo de detección en categorías sugestivas de malignidad disminuyó a nivel nacional, algunos Estados requieren reforzar la aplicación de programas de detección del CaMa mediante mastografía e incrementar la participación de la población blanco.

PALABRAS CLAVE: BIRADS. Mamografía. Sospecha de malignidad. Geolocalización.

\section{Spatio-temporal trend of BIRADS classification suggestive of malignancy: a national analysis of mammograms, 2013-2017}

\section{Abstract}

Introduction: Promoting breast cancer $(B C)$ detection in women by means of mammography is a viable strategy to reduce the number of diagnoses at clinically advanced stages and mortality. Objectives: To describe the results reported by mammography studies in women, carried out nationally during 2013-2017, and to analyze the spatiotemporal trend of Breast Imaging Reporting and Data System (BIRADS) categories suggestive of malignancy by State. Method: Longitudinal, analytical design that included information on mammography studies of women according to age group ( $<40$ and $\geq 40$ ), evaluated in units of the Ministry of Health of Mexico during 2013-2017. The frequency of BIRADS categories and a standardized rate suggestive of malignancy (categories 4 and 5) were estimated in women aged $\geq 40$ years, and spatial statistics were used to analyze the trend by State. Results: $A$ total of 3,659,151 mammograms were analyzed, $98.5 \%$ in women aged $\geq 40$ years. The malignancy-suggestive rate decreased from 38.3 (2013) to 31 (2017) per 100,000 women aged $\geq 40$ years; however, the risk of detection increased up to 13 times in ten States. Conclusions: Although the risk of detection in categories suggestive of malignancy decreased at the national level, some States need to reinforce the application of BC detection programs through mammography and increase the participation of the target population.

KEY WORDS: BIRADS. Mammography. Suspicion for malignancy. Geolocation.

Correspondencia:

*Antonio Reyna-Sevilla

E-mail: gs.antonioreyna@gmail.com

DOI: 10.24875/GMM.20000192
Gac Med Mex. 2021;157:174-180

Disponible en PubMed

www.gacetamedicademexico.com

CC BY-NC-ND (http://creativecommons.org/licenses/by-nc-nd/4.0/). 


\section{Introducción}

El sistema BIRADS (Breast Imaging Reporting and Data System) fue desarrollado por el Colegio Americano de Radiología (ACR); ${ }^{1}$ constituye el lenguaje universal para clasificar los hallazgos en estudios por imagen de los senos a fin de establecer el grado de sospecha de cáncer de mama (CaMa) y la conducta de manejo médico que seguir en cada caso. ${ }^{2}$ En este sentido, el estudio de mastografía reporta una clasificación numérica entre 0 y 6 , cuyos resultados indican: evaluación incompleta que requiere otros estudios complementarios (categoría 0 ), hallazgos no relacionados sospecha de malignidad (categorías 1 y 2), probablemente benigno (categoría 3), con sospecha de malignidad (categorías 4 o 5), o bien malignidad ya demostrada mediante biopsia (categoría 6). ${ }^{3}$ Se reconoce que el método de detección oportuna de CaMa más costo-efectivo es la mastografía, no así la autoexploración y la valoración clínica de senos, ${ }^{4}$ ya que permite detectar oportunamente el padecimiento en fases clínicas iniciales y, en consecuencia, reducir los índices de mortalidad entre el 20 y el $30 \%$ en mujeres > 50 años. ${ }^{5}$ Sin embargo, para lograr este objetivo, la Organización Mundial de la Salud (OMS) aconseja que los programas de tamizaje de detección de CaMa por mastografía alcancen una cobertura o participación del $70 \%$ de la población blanco. ${ }^{6}$

En apego a las recomendaciones y lineamientos de la NOM-041-SSA2-2011 Para la prevención, diagnóstico, tratamiento, control y vigilancia epidemiológica del cáncer de mama, en México se recomienda el estudio de mastografía de tamizaje a toda la población femenina de 40 a 69 años de edad. ${ }^{7}$ No obstante, la Encuesta Nacional de Salud y Nutrición (ENSANUT) reportó en el año 2012 una participación de 36 \% de la población blanco. ${ }^{8}$ Más recientemente, el Instituto Nacional de Estadística y Geografía (INEGI) reportó en 2016 las entidades federativas donde se realizó un mayor número de mastografías: Ciudad de México (29.4\%), Veracruz (6.7\%) y Nuevo León (6.3\%); en contraste, Colima registró solo el $0.4 \% .{ }^{9}$ Dicho panorama, por lo tanto, podría explicar el hecho de que la mayoría de los diagnósticos de CaMa en mujeres mexicanas se realizan en fases clínicamente avanzadas, donde el éxito de los tratamientos oncológicos y las posibilidades de supervivencia se reducen considerablemente. ${ }^{10}$
Actualmente aún son escasos los estudios publicados en México que analicen geográficamente la variabilidad y los cambios de las categorías BIRADS reportadas por estudios de mastografía. Sin embargo, en otros países se han desarrollado estudios epidemiológicos con enfoque geográfico para determinar no solo las zonas donde se requiere mejorar las estrategias de prevención secundaria mediante programas de tamizaje, y que estén asociadas a una detección de categorías con sospecha de malignidad; ${ }^{11-13}$ sino que también es posible identificar el grado de participación de la población blanco y grupos de alto riesgo definidos por edad o lugar de residencia. Así, la evidencia ha contribuido en focalizar geográficamente el mejoramiento de estrategias orientadas a promover la detección temprana de CaMa mediante mastografía y de esta manera reducir eventualmente la carga de diagnósticos en fases clínicamente avanzadas, así como la mortalidad. ${ }^{12}$ Por lo anterior, los objetivos de este estudio fueron, por un lado, describir los resultados reportados por estudios de mastografía realizados en mujeres a nivel nacional en el quinquenio 2013-2017 y, por otro, analizar la tendencia espaciotemporal, por entidad federativa, de aquellas categorías con sospecha de malignidad según la clasificación BIRADS.

\section{Material y métodos}

A partir de datos oficiales reportados por el Centro Nacional de Equidad de Género y Salud Reproductiva (CNEGSR) sobre Cáncer de la Mujer (SICAM), Cáncer de mama,$^{14}$ se llevó a cabo un diseño analítico y longitudinal, con enfoque geográfico, en el que se incluyó información sobre el tamizaje mediante mastografía realizado en mujeres en unidades de la Secretaría de Salud distribuidas en las 32 entidades federativas de México. De dicha fuente se obtuvieron los resultados reportados por estudios de mastografía realizados en el quinquenio 2013-2017, por lo que una primera etapa del análisis consistió en determinar la frecuencia de categorías según la clasificación BIRADS. ${ }^{15}$

Dada la importancia clínica que implican las categorías con sospecha de malignidad, se hizo énfasis en aquellos estudios que reportaron BIRADS 4 y 5 , con la finalidad de realizar un análisis geográfico basado en la estimación de tasas estandarizadas por edad y año. Por lo anterior, dichos estudios fueron georreferenciados por entidad federativa y clasificados en dos grupos: < 40 años e $\geq 40$ años, lo que obedeció a criterios establecidos en la NOM-041-SSA2-2011 
respecto a la edad recomendada para realizarse el estudio de mastografía, ya sea de tamizaje o diagnóstica: ${ }^{7}$ aunado a que el riesgo de CaMa en mujeres aumenta exponencialmente a partir de los 40 años. ${ }^{16}$ En el cálculo de las tasas estandarizadas únicamente se utilizaron los estudios de mastografía que reportaron categorías 4 y 5 (sospecha de malignidad) en mujeres $\geq 40$ años según entidad federativa y año (numerador), así como proyecciones de población femenina reportadas por el Consejo Nacional de Población (CONAPO) en el periodo 2013-2017 (denominador). ${ }^{17}$

A partir de lo anterior, se utilizó regresión lineal para analizar la tendencia temporal respecto a la detección en categorías sospechosas de malignidad en mujeres $\geq 40$ años. Por otro lado, la tendencia espacial se examinó mediante un mapa en el que se mostraron las entidades federativas donde el riesgo aumentó o disminuyó al comparar la tasa estandarizada de 2017 respecto a la registrada en 2013. Por último, se utilizó la técnica de interpolación espacial ${ }^{18}$ para generar una superficie (modelo) a lo largo del país en la que, a partir de líneas, se unieron valores similares asociados a la tasa estandarizada en el quinquenio 20132017. Esto se hizo con la finalidad de visualizar áreas en las que podrían detectarse mastografías con sospecha de malignidad en mujeres $\geq 40$ años a nivel municipal, incluso cuando no fue posible obtener la información de estudios mastográficos en dicha escala territorial, dado que una de las contribuciones de la técnica de interpolación espacial fue estimar dicho riesgo por municipio. ${ }^{18} \mathrm{El}$ análisis de los datos se realizó con el programa SPSS versión 23 y los mapas temáticos en QGIS versión 3.10.

Este estudio se clasificó como una investigación sin riesgo, de acuerdo con la Ley General de Salud en Materia de Investigación para la Salud en México. Los datos obtenidos del registro público se manejaron en apego a las pautas éticas del Consejo de Organizaciones Internacionales de las Ciencias Médicas (CIOMS, por sus siglas en inglés), pauta 22 , sobre el uso de datos obtenidos en línea y de herramientas digitales en investigaciones relacionadas con la salud..$^{19}$

\section{Resultados}

De acuerdo con la fuente consultada para realizar el análisis descriptivo y geográfico, en el quinquenio 2013-2017 se realizaron 3,659,151 mastografías, de las cuales el $98.5 \%(n=3,603,586)$ correspondió a mujeres $\geq 40$ años (media $[\bar{x}]=50.7$, desviación

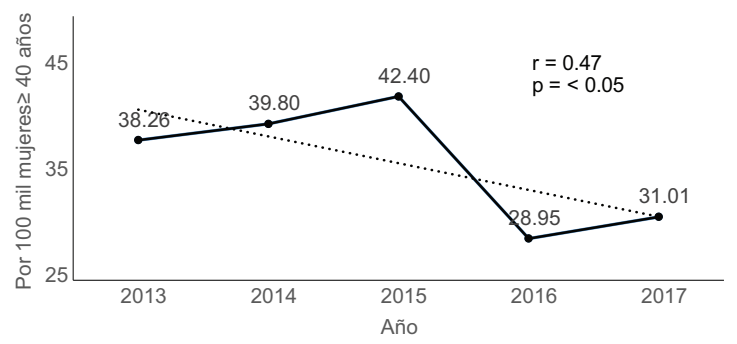

Figura 1. Tendencia de detección en categorías con sospecha de malignidad (BIRADS 4 y 5) en mujeres $\geq 40$ años reportadas por estudios de mastografía en México, $2013-2017$ (elaboración de los autores a partir de datos reportados por el Centro Nacional de Equidad de Género y Salud Reproductiva sobre Cáncer de la Mujer $\left.{ }^{14}\right)$. BIRADS = Breast Imaging Reporting and Data System .

Tabla 1. Frecuencia relativa de categorías BIRADS reportadas por estudios de mastografía en mujeres según grupo de edad, México 2013-2017

\begin{tabular}{l|c|c|}
\hline \multirow{2}{*}{ Categorías según BIRADS } & \multicolumn{2}{|c|}{ Grupo de edad } \\
\cline { 2 - 3 } & $<40$ años & $\geq 40$ años \\
\cline { 2 - 3 } & $\overline{\mathrm{x}}=36.8 \mathrm{DE}=\mathbf{2 . 9}$ & $\overline{\mathrm{x}}=50.7 \mathrm{DE}=7.4$ \\
\hline Incompleta (0) & 10.18 & 7.65 \\
\hline Negativa (1) & 19.67 & 19.26 \\
\hline Benigna (2) & 60.84 & 67.43 \\
\hline Probablemente benigna (3) & 5.77 & 4.64 \\
\hline Sospechosa (4) & 2.39 & 0.75 \\
\hline $\begin{array}{l}\text { Altamente sugestiva de } \\
\text { malignidad (5) }\end{array}$ & 1.11 & 0.23 \\
\hline SD & 0.012 & 0.0006 \\
\hline$\%$ & 1.50 & 98.5
\end{tabular}

BIRADS = Breast Imaging Reporting and Data System; $\overline{\mathrm{x}}=$ media; $\mathrm{DE}=$ desviación estándar; $\mathrm{SD}=$ sin definir.

Elaboración de los autores a partir de datos reportados por el Centro Nacional de Equidad de Género y Salud Reproductiva (CNEGSR) sobre Cáncer de la Mujer $(\text { SICAM })^{14}$.

estándar $[\mathrm{DE}]=7.4$ ), esto es, aproximadamente el $25 \%$ de la población femenina (entre 40 y 69 años) que residía en México durante el mismo periodo. En la Tabla 1 se presenta la frecuencia relativa de las categorías BIRADS reportadas según grupo de edad; el $86.7 \%(n=3,124,487)$ se clasificó como negativo o benigno en mujeres $\geq 40$ años.

Por otra parte, la probabilidad de detección con sospecha de malignidad (BIRADS 4 y 5) mostró una tendencia descendente $(p<0.05)$; la tasa estandarizada a nivel nacional cambió de 38.3 (2013) a 31 (2017) por 100 mil mujeres $\geq 40$ años (Figura 1). Sin 


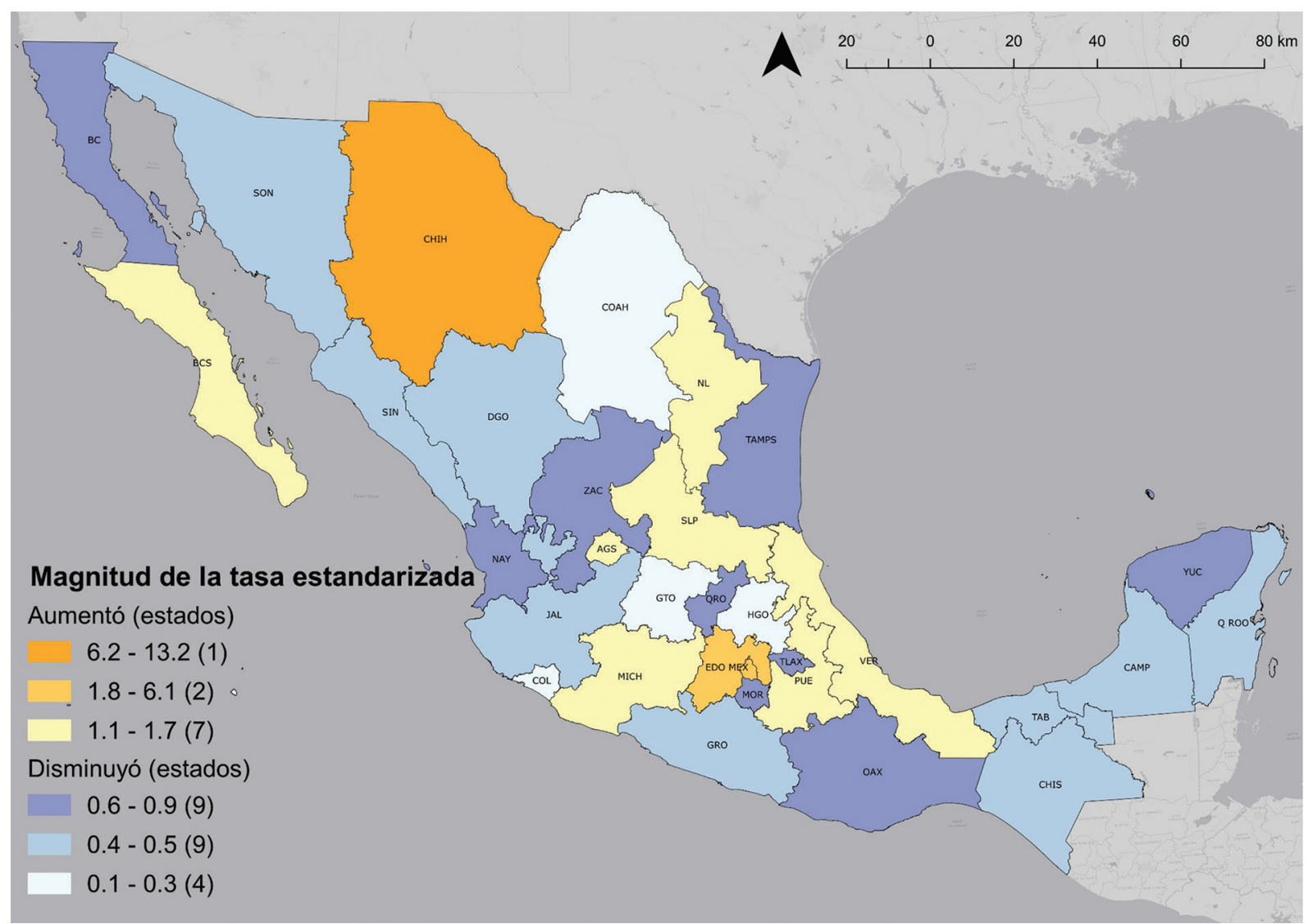

Figura 2. Variación geográfica de la tasa estandarizada de categorías con sospecha de malignidad (BIRADS 4 y 5) en mujeres $\geq 40$ años, comparación entre 2013 y 2017 (elaboración de los autores a partir de datos reportados por el Centro Nacional de Equidad de Género y Salud Reproductiva sobre Cáncer de la Mujer $\left.{ }^{14}\right)$. BIRADS = Breast Imaging Reporting and Data System.

embargo, al comparar la tasa de 2017 respecto a la registrada en 2013 se identificaron 10 (31.3\%) Estados, localizados principalmente en el centro y norte del país (patrón regional), en los cuales el riesgo de detección en categorías 4 y 5 aumentó hasta 13 veces; por ejemplo, en Chihuahua la tasa cambió de 5.8 (2013) a 76.1 (2017) por 100 mil mujeres $\geq 40$ años y en la Ciudad de México la variación fue de 7.4 (2013) a 44.8 (2017) por 100 mil mujeres $\geq 40$ años (Figura 2). Adicionalmente, los Estados donde se registró un mayor riesgo de detección con sospecha de malignidad en el quinquenio fueron Campeche y Coahuila, cuya tasa estandarizada fue 68.3 y 76.4 por 100 mil mujeres $\geq 40$ años, respectivamente (Figura 3 ).

Respecto al modelo espacial representado en la Figura 4, se identificaron dos zonas en las que, en comparación con otros Estados, se podría detectar un mayor número de mastografías con sospecha de malignidad; una localizada al sureste del país, que involucra municipios de Tabasco y Campeche, y otra que va desde el occidente (Jalisco, Aguascalientes,
Guanajuato, Querétaro y Zacatecas) hasta el norte de México (Nuevo León, Coahuila y Chihuahua).

\section{Discusión}

Aun cuando 8 de cada $10(n=3,169,229)$ estudios de mastografía reportaron BIRADS 102 , y la tendencia de detección con sospecha de malignidad disminuyó a nivel nacional, al comparar la tasa estandarizada del año 2017 respecto a la de 2013 el riesgo aumentó considerablemente en 10 (31.3\%) Estados. Por lo tanto, la evidencia destaca la importancia que implica continuar y mejorar los programas de tamizaje mediante mastografía en México, por un lado, porque estos están siendo utilizados como método de detección temprana y no como parte de la evaluación diagnóstica, por otro, en algunos Estados ha aumentado hasta 13 veces el riesgo de detección en BIRADS 4 y 5 . Sin embargo, otro aspecto que destacar a partir de los resultados es la importancia de incrementar la participación de las mujeres en el proceso de detección temprana del CaMa. 


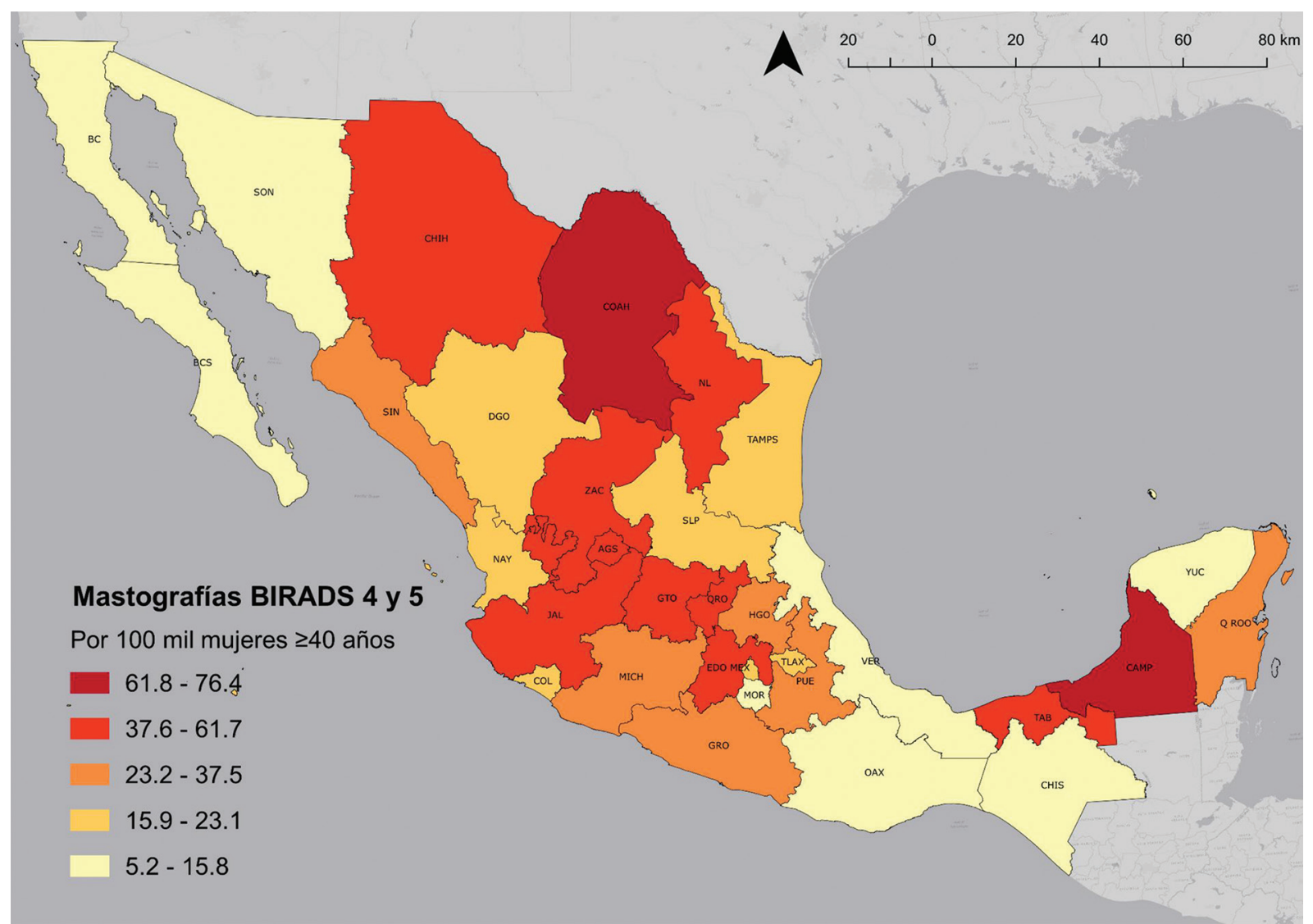

Figura 3. Tasa estandarizada de detección en categorías con sospecha de malignidad (BIRADS 4 y 5) en mujeres $\geq 40$ años por entidad federativa, 2013-2017 (elaboración de los autores a partir de datos reportados por el Centro Nacional de Equidad de Género y Salud Reproductiva sobre Cáncer de la Mujer ${ }^{14}$ ). BIRADS = Breast Imaging Reporting and Data System.

Si bien durante el quinquenio 2013-2017 se encontró una disminución en la probabilidad de detección en categorías con sospecha de malignidad de CaMa a nivel nacional, también se identificaron algunos Estados donde se requiere reforzar las estrategias establecidas en la NOM-041-SSA2-2011, en términos de vigilancia epidemiológica y detección oportuna. ${ }^{7}$ En efecto, Ciudad de México, Estado de México, Michoacán y Chihuahua registraron una tasa entre 42.4 y 76.1 por 100 mil mujeres $\geq 40$ años en el 2017, además, según el modelo espacial presentado, en Chihuahua es posible que continúe la detección de mastografías en BIRADS 4 y 5 (Figura 4). Por lo anterior, la evidencia presentada contribuye en priorizar aquellos Estados donde es necesario intensificar la prevención secundaria mediante la realización de estudios de mastografía a nivel poblacional. Tal sería el caso de Chihuahua, que según estadísticas reportadas por el INEGI en $2016^{\circ}$ fue de los Estados con mayor tasa de mortalidad por CaMa (20 y 26 por 100 mil mujeres). Por tanto, se trata de una alternativa para disminuir la cantidad de resultados clasificados con sospecha de malignidad y los diagnósticos en fases clínicamente avanzadas. ${ }^{5}$

En los últimos 10 años en México se ha reportado una limitada participación de mujeres en la realización de mastografías como lo establece la NOM-041SSA2-2011, ${ }^{7}$ esto es, entre el $11.7^{8}$ y el $20 \% .^{10}$ Aunque no se cuenta con evidencia suficiente, derivada del análisis geográfico sobre estudios de mastografía, es probable que solo el $25 \%$ de la población blanco tuviera como antecedente un estudio de esta naturaleza, lo que exhibe un parámetro inferior al recomendado por la OMS a fin de reducir la mortalidad por CaMa hasta en un $30 \% .^{5}$ En este contexto, la identificación de Estados prioritarios a nivel nacional, centro y norte del país (Figura 2) adquiere importancia con el propósito de definir en dónde es necesario no solo promover la detección oportuna de $\mathrm{CaMa}$ mediante mastografía, como se ha realizado en otros países, ${ }^{11-13}$ sino también aumentar la participación de las mujeres; la mayoría de los estudios mastográficos 


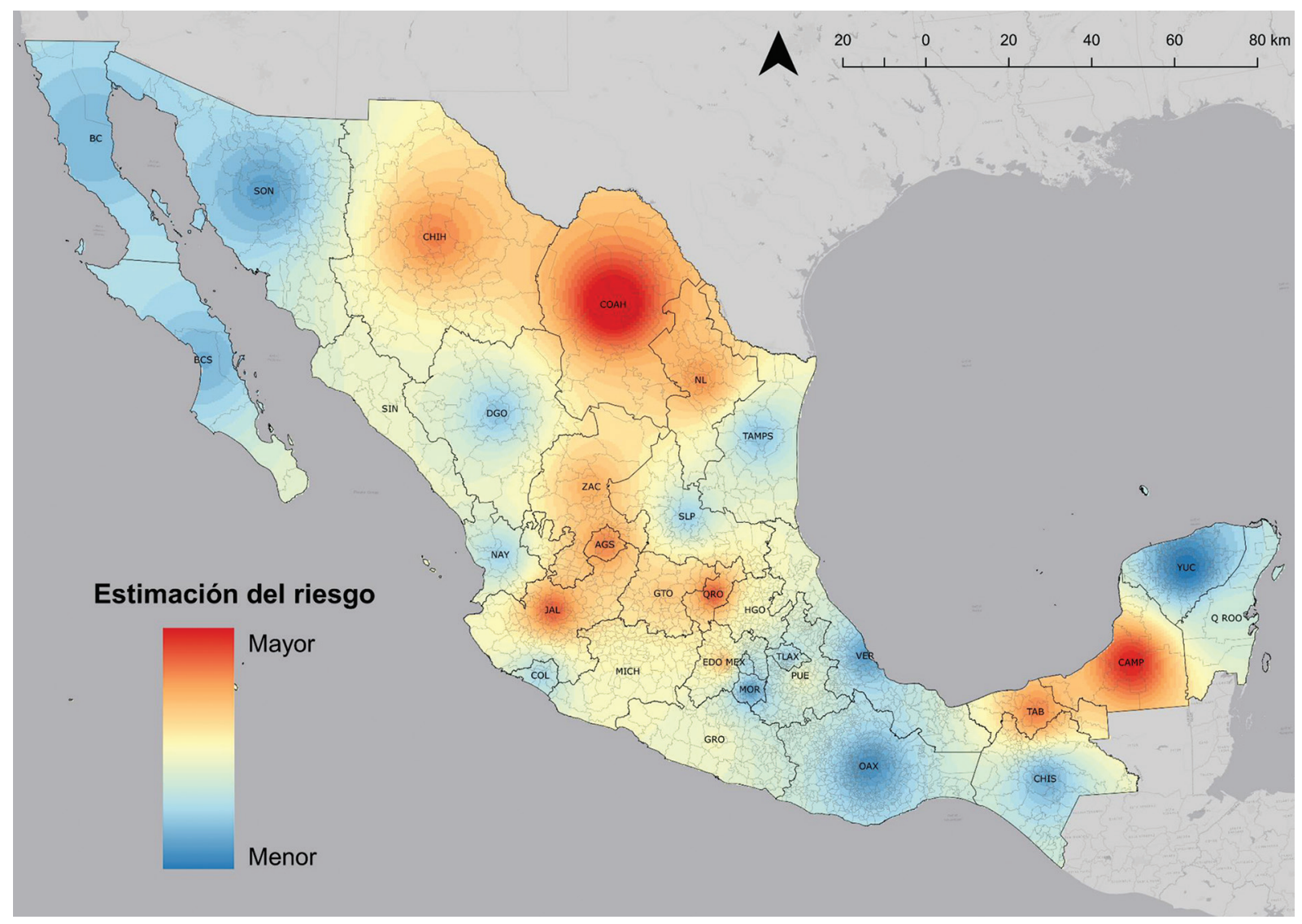

Figura 4. Modelo espacial de detección de mastografías en categorías con sospecha de malignidad (BIRADS 4 y 5) en mujeres $\geq 40$ años por municipio y entidad federativa (elaboración de los autores a partir de datos reportados por el Centro Nacional de Equidad de Género y Salud Reproductiva sobre Cáncer de la Mujer ${ }^{14}$ ). BIRADS = Breast Imaging Reporting and Data System.

ha estado reportando resultados favorables, esto es, categorías 1 y 2 según la clasificación BIRADS. ${ }^{15}$

Ahora bien, es necesario mencionar algunas limitaciones intrínsecas al diseño metodológico presentado. El nivel de desagregación territorial establecido fue por entidad federativa, y ello limitó analizar el comportamiento espacio-temporal a nivel municipal con la finalidad de obtener resultados focalizados. Por otra parte, la base de datos utilizada no permitió determinar cuántas mujeres se realizaron mastografías en dos o más ocasiones en el periodo de estudio, por lo que esto podría disminuir el porcentaje real de participación en el proceso de detección de CaMa. Tampoco se reportó si el estudio de mastografía fue con fines de tamizaje o diagnóstico, como establece la NOM-041-SSA2-201, ${ }^{7}$ lo que resulta ser de especial interés en el seguimiento y evaluación de dicho proceso. Sin embargo, hay algunas fortalezas que destacar, esto es, el análisis permitió comparar al mismo tiempo la tendencia temporal (en el quinquenio 2013-2017) y el comportamiento espacial (considerando las 32 entidades federativas) de la detección de CaMa mediante mastografías, así como visualizar un indicador de riesgo a nivel municipal. De acuerdo con los autores, este estudio representa el análisis más reciente, realizado a nivel nacional y estatal, sobre los resultados reportados por estudios de mastografía en mujeres mexicanas; la cantidad de datos analizados implica una muestra significativa $\sin$ estudios precedentes de esta naturaleza.

En conclusión, del total de estudios mastográficos analizados en el quinquenio 2013-2017, el 98 \% se realizó en mujeres $\geq 40$ años y 8 de cada 10 reportaron categorías 1 o 2 según BIRADS, lo cual está en apego con la NOM-041-SSA2-2011 respecto a realizar el examen en este grupo poblacional y como método de detección temprana. ${ }^{7}$ Sin embargo, aunque la proporción de Estados en los que aumentó la probabilidad de detección en las categorías 4 y 5 fue del $31.3 \%$ (centro y norte), ello implicó una magnitud hasta 13 veces mayor en 2017 respecto al año 2013. 
Así, la evidencia sugiere mejorar o reforzar los programas de detección de CaMa mediante mastografía (prevención secundaria), principalmente en Estados con población en riesgo o cuya tendencia de detección en etapas con sospecha de malignidad ha ido aumentando (centro y norte), a fin de encontrar las lesiones en etapas oportunas como ha sucedido recientemente en la mayoría de los Estados en México. No obstante, también se requiere incrementar el número de mujeres con antecedente de mastografía a partir de los 40 años, dado que ello representa una estrategia útil en el control y vigilancia epidemiológica del CaMa para reducir no solo los diagnósticos en fases avanzadas, sino también la mortalidad, ambos considerados problemas vigentes de salud pública en México.

\section{Agradecimientos}

A las mujeres que se realizaron su estudio mastográfico durante el quinquenio 2013-2017, así como al personal de salud involucrado en la generación y actualización de la base de datos utilizada en este estudio epidemiológico.

\section{Conflicto de intereses}

Los autores declaran no tener conflicto de intereses alguno.

\section{Financiamiento}

Los autores no recibieron patrocinio para llevar a cabo este artículo.

\section{Responsabilidades éticas}

Protección de personas y animales. Los autores declaran que para esta investigación no se han realizado experimentos en seres humanos ni en animales.

Confidencialidad de los datos. Los autores declaran que han seguido los protocolos de su centro de trabajo sobre la publicación de datos de pacientes.

Derecho a la privacidad y consentimiento informado. Los autores han obtenido el consentimiento informado de los pacientes y/o sujetos referidos en el artículo. Este documento obra en poder del autor de correspondencia.

\section{Bibliografía}

1. D'Orsi CJ, Sickles EA, Mendelson EB, Morris EA, et al. ACR BIRADS $^{\circledR}$ Atlas, Breast Imaging Reporting and Data System. Reston, VA, American College of Radiology; 2013.

2. Torres Tabanera M. Novedades de la $5 .^{a}$ edición del sistema breast imaging reporting and data. Rev Senol y Patol Mamar. 2016;29(1):32-9.

3. Como entender su informe de mamograma [Internet]. American Cancer Society; 2019 [consultado: enero 2020]. Disponible en: https://www.cancer.org/es/cancer/cancer-de-seno/pruebas-de-deteccion-y-deteccion-temprana-del-cancer-de-seno/mamogramas/como-entender-su-informe-de-mamograma.html

4. WHO position paper on mammography screening [Internet]. World Health Organization; 2014 [consultado: enero 2020]. Disponible en: http:// apps.who.int/iris/bitstream/handle/10665/137339/9789241507936_eng. pdf; isessionid=9294040BE7603C4B8AD2F1FDECAFF350? sequence $=1$

5. Cáncer de mama: prevención y control. [Internet]. Organización Mundial de la Salud; 2020 [consultado: enero 2020]. Disponible en: https://www. who.int/topics/cancer/breastcancer/es/

6. Detección temprana. Control del Cáncer: aplicación de los conocimientos. Guía de la OMS para desarrollar programas eficaces [Internet]. Organización Mundial de la Salud; 2007 [consultado: enero 2020]. Disponible en: https://www.paho.org/hq/index.php?option=com_topics\&view $=$ rdmore \&cid=3681\&ltemid $=40735 \&$ lang $=e s$

7. Norma Oficial Mexicana NOM-041-SSA2-2011, Para la prevención, diagnóstico, tratamiento, control y vigilancia epidemiológica del cáncer de mama [Internet]. México: Secretaría de Salud, Diario Oficial de la Federación; 2011 [consultado: enero 2020]. Disponible en: http://dof.gob.mx/ nota detalle.php?codigo $=5194157 \&$ fecha $=09 / 06 / 2011$

8. Encuesta Nacional de Salud y Nutrición, ENSANUT [Internet]. Cuernavaca, México: Instituto Nacional de Salud Pública; 2012 [consultado: enero 2020]. Disponible en: http://ensanut.insp.mx

9. Estadísticas a propósito del día mundial de la lucha contra el cáncer de mama (19 de octubre) [Internet]. México: Instituto Nacional de Estadística y Geografía; 2016 [consultado: febrero 2020]. Disponible en: https:// www.ues.mx/Docs/avisos/diamundialcancerdemama.pdf

10. Cabrera-Galeana P, Soto-Perez-de-Celis E, Reynoso-Noverón N, Villarreal-Garza C, Arce-Salinas C, Matus-Santos J, et al. Clinical characteristics and outcomes of older women with breast cancer in Mexico. $J$ Geriatr Oncol. 2018;9(6):620-5.

11. Schootman M, Jeff DB, Gillanders WE, Yan Y, Jenkins B, Aft R. Geographic clustering of adequate diagnostic follow-up after abnormal screening results for breast cancer among low-income women in Missouri. Ann Epidemiol. 2007;17(9):704-12.

12. Sirous M, Shahnani PS, Sirous A. Investigation of frequency distribution of Breast Imaging Reporting and Data System (BIRADS) classification and epidemiological factors related to breast cancer in Iran: A 7-year study (2010-2016). Adv Biomed Res. 2018;7:56.

13. Sánchez-Hernández AG, Ortiz-Mendoza CM. Frecuencia de uso de la mastografía por derechohabientes del ISSSTE en un hospital general urbano. Gac Mex Oncol. 2019;18(3):127-30.

14. Datos abiertos sobre cáncer de la mujer (SICAM). Cáncer de mama [Internet]. México: Centro Nacional de Equidad de Género y Salud Reproductiva; 2020 [consultado: diciembre 2019]. Disponible en: http:// cnegsr.salud.gob.mx

15. Camacho-Piedra C, Espíndola-Zarazúa V. Actualización de la nomenclatura BI-RADS $®$ por mastografía y ultrasonido. Rev An Radiol México. 2019;17(2):100-8.

16. Sánchez J, Valle-Solís A, Arce-Salinas C, Bargalló-Rocha JE, Bautista-Piña V, Cervantes-Sánches G, et al. Consenso Mexicano sobre diagnóstico y tratamiento del cáncer mamario. Octava revisión. Colima 2019. Gac Mex Oncol. 2018;18:141-231.

17. Proyecciones de la Población de México y de las Entidades Federativas, 2016-2050 [Internet]. México: Consejo Nacional de Población; 2020 [consultado: diciembre 2019]. Disponible en: https://datos.gob.mx/busca/dataset/proyecciones-de-la-poblacion-de-mexico-y-de-las-entidades-federativas-2016-2050

18. Auchincloss AH, Gebreab SY, Mair C, Diez Roux AV. A review of spatial methods in epidemiology, 2000-2010. Annu Rev Publ Health. 2012;33:107-22.

19. Organización Panamericana de la Salud y Consejo de Organizaciones Internacionales de las Ciencias Médica. Pautas éticas internacionales para la investigación relacionada con la salud con seres humanos, Cuarta Edición. Ginebra: Consejo de Organizaciones Internacionales de las Ciencias Médicas; 2016. 